\title{
Percutaneous sonography-guided radiofrequency ablation in the management of parathyroid adenoma
}

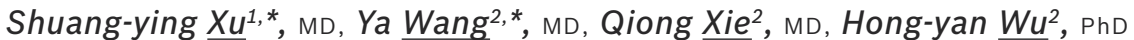

\begin{abstract}
Parathyroid adenoma is a major cause of primary hyperparathyroidism. Treatment usually involves the surgical removal of one or more parathyroid glands. However, specific localising techniques have boosted the development of nonsurgical, minimally invasive procedures, such as percutaneous radiofrequency ablation (RFA) under ultrasonographic guidance, which are gaining popularity as a method of treatment. This paper reports two male patients who presented with hypercalcaemia (highest serum calcium level $4.56 \mathrm{mmol} / \mathrm{L}$ and $4.57 \mathrm{mmol} / \mathrm{L}$, respectively) and hyperparathyroidism (highest serum parathyroid hormone [PTH] level $772 \mathrm{ng} / \mathrm{L}$ and $>1,900 \mathrm{ng} / \mathrm{L}$, respectively) due to solitary parathyroid adenoma. Four days after percutaneous RFA, the serum calcium levels in both patients decreased and PTH levels showed a significant decreasing trend. Both patients recovered well with normal levels of calcium and improvement of symptoms. Thus, we conclude that RFA may be a safe and effective therapeutic option in the treatment of parathyroid adenoma.
\end{abstract}

Keywords: hypercalcaemia, intervention, parathyroid adenoma, radiofrequency ablation

\section{INTRODUCTION}

Parathyroid adenoma is a major cause of primary hyperparathyroidism, with an incidence of $80 \%-85 \%$. $^{(1)}$ Currently, the primary method of treatment is parathyroidectomy. ${ }^{(2,3)}$ This method of treatment, however, may not be possible when there is a high surgical risk or contraindication for surgery, or when the patient is reticent to undergo surgical procedures. The latter being a concern raised mainly by women due to the possibility of a resulting postoperative scar. Hence, in most cases, parathyroidectomy is only performed on selected patients.

With the swift development of minimally invasive and highly specific techniques, percutaneous sonography-guided radiofrequency ablation (RFA) therapy has been steadily progressing and yielding good clinical results. ${ }^{(4-7)}$ While RFA has been used as a reasonably effective method to treat some primary and secondary tumours of the thyroid, ${ }^{(4)}$ lung $^{(5,6)}$ and liver, ${ }^{(7)}$ the procedure is not limited to these organs. This report describes our experience with RFA in the treatment of parathyroid adenoma, and aims to assess the safety, effectiveness and durability of this procedure for the treatment of parathyroid adenoma.

\section{CASE REPORTS}

\section{Case 1}

A 50-year-old man presented to our hospital with a onemonth history of vomiting, general fatigue and bone pain. The results of the laboratory investigations revealed elevated serum parathyroid hormone (PTH) (761-772 ng/L; normal range $[N R]<72 \mathrm{ng} / \mathrm{L})$, elevated serum calcium

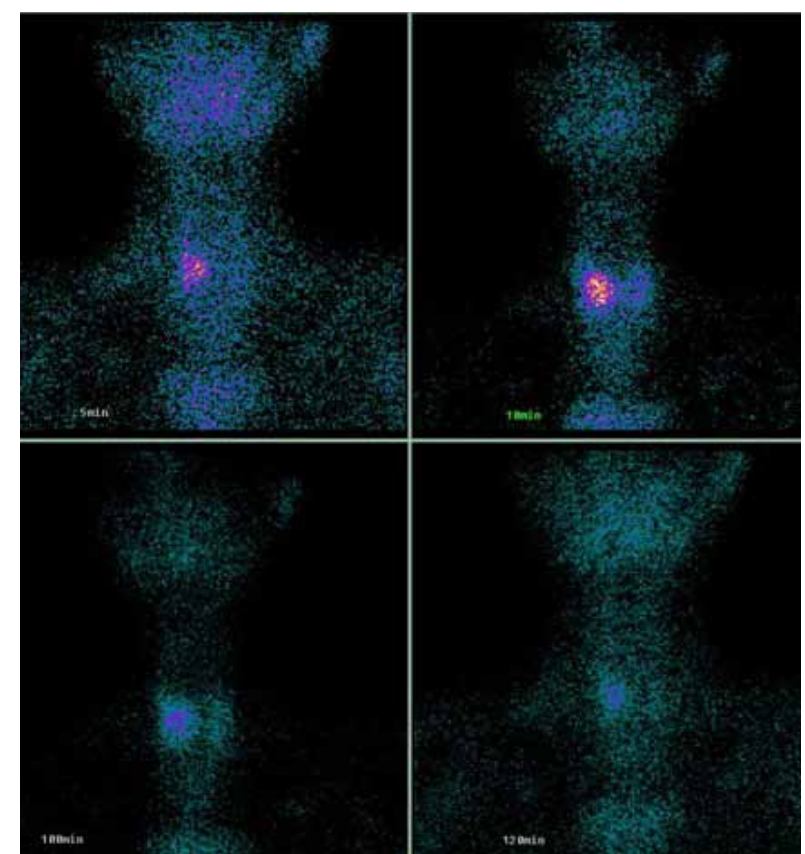

Fig. 1 Case 1: ${ }^{99 m}$ Tc-sestamibi scintigraphy images before radiofrequency ablation show increased uptake of the radionuclide in the middle right lobe of the thyroid gland.

(3.99-4.56 mmol/L; NR 2.00-2.65 mmol/L), and low serum phosphate $(0.59 \mathrm{mmol} / \mathrm{L} ; \mathrm{NR} 0.8-1.5 \mathrm{mmol} / \mathrm{L})$ levels. Markedly elevated 24-hour urine calcium $(78.1 \mathrm{mmol} / 24 \mathrm{hr}$; NR 2.5-7.5 mmol/24hr) and elevated 24-hour urine phosphate (56.2 mmol/24hr; NR 23.0-48.0 mmol/24hr) levels were observed. Symptoms at presentation also included urinary tract stones, osteoporosis and gastritis. The patient had no prior history of any kind of radiotherapy treatment or endocrine neoplasia.

\footnotetext{
${ }^{1}$ Department of Orthopaedics, ${ }^{2}$ Department of Endocrinology, Jingzhou First People's Hospital, Yangtze University, Hubei, China *These two authors contributed equally as first author in this work.

Correspondence: Dr Hong-yan Wu, Professor and Head, Department of Endocrinology, Jingzhou First People's Hospital, Yangtze University, 8 Hang-Kong Street, Jingzhou, Hubei 434000, China. hongyan_wu2003@yahoo.com.cn
} 

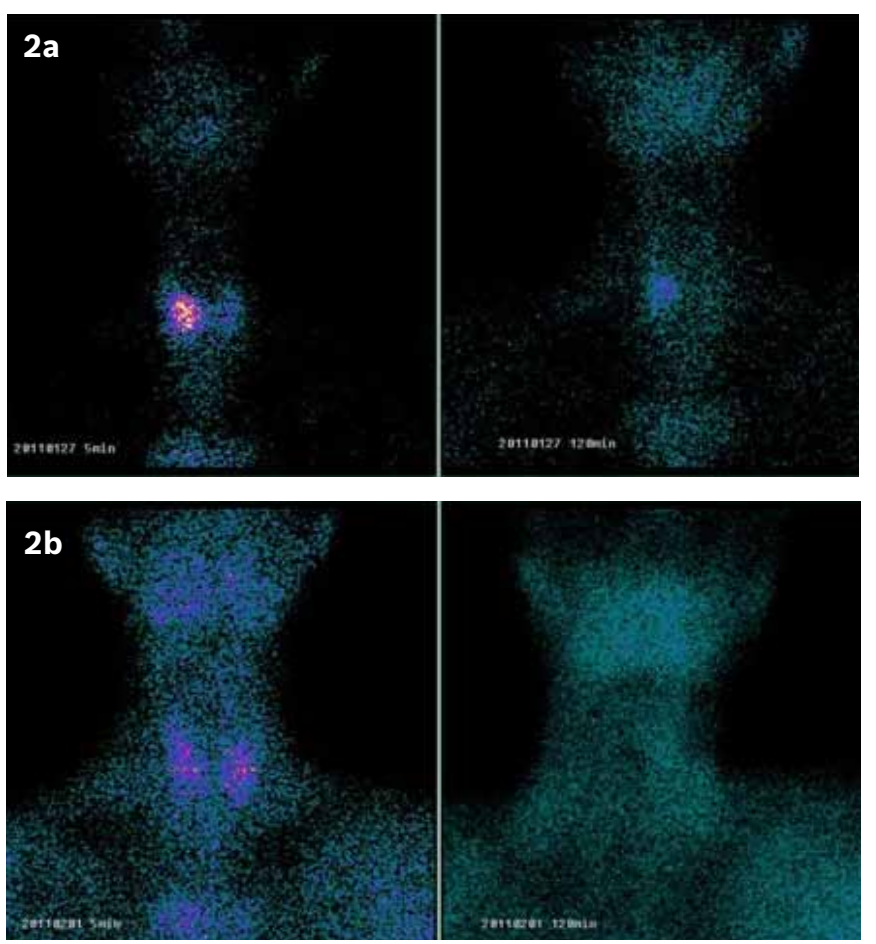

Fig. 2 Case 1: ${ }^{99 \mathrm{~m}} \mathrm{Tc}$-sestamibi scintigraphy images show the difference in uptake (a) pre- and (b) post-treatment with radiofrequency ablation.

Colour Doppler ultrasonography of the neck suggested the presence of a parathyroid adenoma in the right side. Magnetic resonance (MR) imaging of the neck revealed a single adenoma measuring $11.37 \mathrm{~mm} \times 10.09 \mathrm{~mm} \times 15.06 \mathrm{~mm}$ in the right parathyroid gland, with normal mediastinum. Technetium-99m ${ }^{99 \mathrm{~m} T \mathrm{Tc})-s e s t a m i b i}$ scintigraphy revealed an increased uptake of the radionuclide at the right middle lobe of the thyroid gland (Fig. 1). This, together with our clinical findings, was indicative of hyperparathyroidism.

On Day 5 of admission, the patient underwent RFA for the purpose of decreasing his serum calcium and PTH levels, as well as to reduce the signs and symptoms associated with hyperparathyroidism. After local anaesthesia was administered, an isolation belt around the inferior right of the parathyroid gland was created using normal saline to protect the gland from unnecessary injury. A biopsy needle (PRECISA ${ }^{\mathrm{TM}}$; HS Hospital Service SpA, Latina, Italy) was then inserted into the adenomatous tissue under ultrasonographic guidance, and a $2-\mathrm{cm}$ tissue strip was removed for pathologic examination. Subsequently, a radiofrequency generator (Celonlab ENT system, Celon AG, Germany) with an internal needle electrode was used for ablation of the adenomatous tissue. The radiofrequency needle was inserted into the centre of the parathyroid adenomatous tissue, and an ablation power of $5 \mathrm{~W}$ was applied for $12 \mathrm{~min}$. The skin of the neck was not incised, thus preventing unnecessary scar formation. The entire RFA procedure was well tolerated by the patient.

After ablation, the vital signs of the patient were stable and he recovered well. His serum PTH and calcium levels decreased to $234 \mathrm{ng} / \mathrm{L}$ and $2.33 \mathrm{mmol} / \mathrm{L}$, respectively, and his serum phosphate level increased to $0.71 \mathrm{mmol} / \mathrm{L}$, which was still slightly lower than the normal range. Although there was transient hoarseness after the procedure, the patient spontaneously improved two days post procedure. Pathologic findings confirmed the diagnosis of parathyroid adenoma.

${ }^{99 \mathrm{~m} T C}$-sestamibi scintigraphy performed after ablation revealed no abnormal uptake. Fig. 2 shows the difference in uptake pre- and post procedure. Four days after the procedure, the patient presented with hypocalcaemia $(1.84 \mathrm{mmol} / \mathrm{L})$, which was resolved $(2.68 \mathrm{mmol} / \mathrm{L})$ within two weeks with the use of calcium replacement therapy. Two months after the procedure, the patient's serum levels of PTH, calcium and phosphate had normalised, and there was a reduction in his gastrointestinal and bone disease symptoms.

\section{Case 2}

A 54-year-old man with a ten-year history of type 2 diabetes mellitus and a two-week history of general fatigue and bone pain was admitted to our hospital. He had a markedly elevated PTH level (> 1,900 ng/L) and hypercalcaemia (3.79-4.57 mmol/L). Laboratory tests showed a fasting plasma glucose level of $7.90 \mathrm{mmol} / \mathrm{L}$ (NR 3.90-6.10 mmol/L) and a postprandial plasma glucose level of $9.80 \mathrm{mmol} / \mathrm{L}$ (NR 7.80-11.10 mmol/L). Symptoms at presentation included osteoporosis and nephrolithiasis. The patient had no prior history of any kind of radiotherapy treatment or endocrine neoplasia. Colour Doppler ultrasonography of the neck detected a large hypoechoic mass dorsal to the left inferior pole of the thyroid gland, possibly originating from the parathyroid gland. Subsequent computed tomography (CT) of the neck revealed a large oval mass, measuring $26.78 \mathrm{~mm} \times 22.40 \mathrm{~mm} \times 4.90 \mathrm{~mm}$, in the left parathyroid gland (Fig. 3).

On Day 2 of admission, the parathyroid adenoma was treated successfully using RFA under ultrasonographic guidance. The ablation power and time were $5 \mathrm{~W}$ and $30 \mathrm{~min}$, respectively. As in Case 1, a PRECISA ${ }^{\text {TM }}$ biopsy needle was used to remove a 2-cm tissue strip for pathologic examination prior to the RFA procedure. The RFA procedure was well tolerated by the patient. On Day 1 post procedure, the patient's serum PTH and calcium levels decreased to $995 \mathrm{ng} / \mathrm{L}$ and $3.42 \mathrm{mmol} / \mathrm{L}$, respectively. His calcium and blood glucose levels were within the normal range three days after the procedure and his serum PTH level showed further decrease (549 ng/L) two weeks after the procedure. Pathologic findings were in conformity with the imaging results, and the diagnosis of parathyroid adenoma was thus confirmed. At two months after the procedure, the patient's serum calcium level remained normal while his serum PTH level continued to decrease, as evidenced by the reduction of symptoms experienced by the patient. 

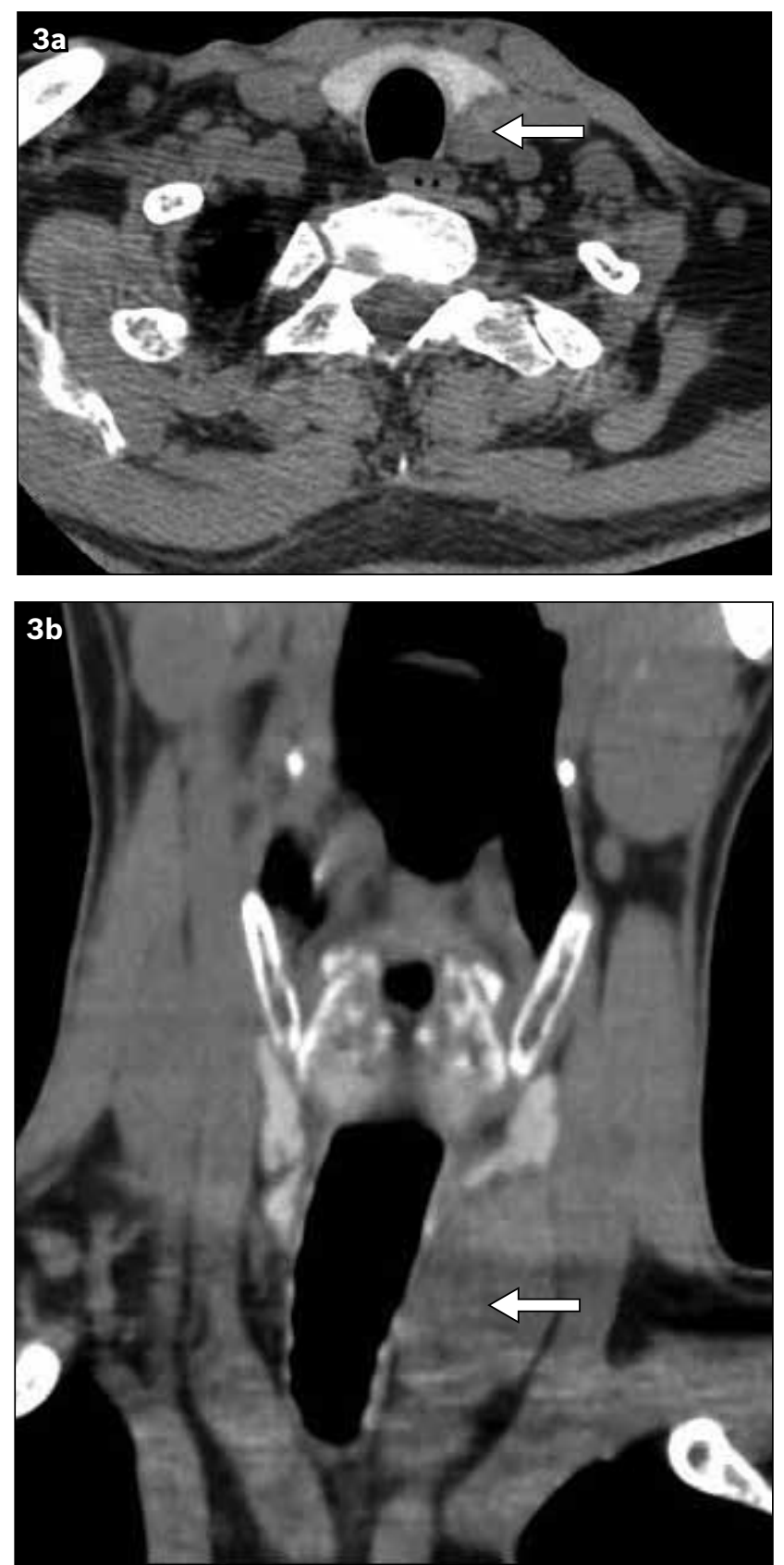

Fig. 3 Case 2: CT images of the patient's neck in the (a) coronal plane and (b) sagittal plane show a large oval mass (arrows) in the left parathyroid gland.

\section{DISCUSSION}

Surgical treatment is currently the main therapeutic option for the management of parathyroid adenoma. While surgery is curative, its drawbacks include the risks associated with the use of general anaesthesia, the need for long incision, and possible recurrent laryngeal nerve injury and scar formation.

Specific localising techniques have boosted the development of nonsurgical, minimally invasive procedures such as ethanol ablation, RFA and laser thermal ablation. ${ }^{(8-10)}$ Evidence in the form of higher survival rates, better local control, lower recurrence rates and complete radiological tumour response supports RFA as a procedure superior to ethanol ablation. ${ }^{(11,12)}$ Furthermore, ethanol ablation requires periodic re-injections in order to achieve a complete cure. This increases the risk of complications such as haemorrhage, transient alcohol intoxication, local pain and tumour cell seeding. Laser thermal ablation is not commonly used due to the high cost of the sophisticated equipment. As such, percutaneous sonography-guided RFA therapy was selected in the two cases described.

In our study, one of the two patients presented with transient hoarseness after RFA, but this was spontaneously resolved two days after the procedure. Beyond that, no other complications were observed and the whole RFA procedure was well tolerated. RFA led to necrosis of the adenoma tissue, resulting in the decrease of the patients' serum calcium level within four days after the procedure. Serum PTH levels for both patients showed a significant decreasing trend post procedure. However, long-term monitoring of serum calcium and PTH levels is important in order to establish the effectiveness of RFA as a therapeutic option for the management of parathyroid adenoma. Thus, RFA therapy is a reasonably effective method for treating parathyroid adenoma, with favourable cosmetic results and fewer drawbacks as compared to surgical treatment, although it may require long-term follow-up. RFA may be an optimal alternative to surgery, especially for patients with high surgical risks, contraindications to surgery due to advanced age, acute severe hypercalcaemia or persistent symptomatic hyperparathyroidism.

In general, adequate and concordant localisation of the adenomatous tissue before RFA is needed to ensure that the procedure is safe and minimally invasive. Colour Doppler ultrasonography is a useful and accessible technique for the diagnosis of cervical adenomas, ${ }^{(13)}$ and ${ }^{99 \mathrm{~m} T c-s e s t a m i b i}$ scintigraphy is the method of choice for localisation of parathyroid adenomas due to its high diagnostic sensitivity (> 90\%). ${ }^{(14)} \mathrm{CT}$ and MR imaging are helpful in the diagnosis and further delineation of the location of the adenomatous tissue. ${ }^{(15)}$ The two patients in our study were subject to a mixture of the aforementioned localisation techniques so as to improve the accuracy of diagnosis and localisation of the parathyroid adenoma.

As parathyroid adenoma is a rare disease, data from large retrospective studies and randomised trials for the verification of therapeutic options are limited. Therefore, physicians may have to make management decisions based on evidence from case reports while further evaluating the therapeutic efficacy of RFA through long-term accumulation of experience.

In conclusion, the use of ultrasonography-guided percutaneous RFA in the management of parathyroid adenoma is safe and yields a high degree of recovery from hypercalcaemia. As RFA is an effective therapeutic option for the management of parathyroid adenoma, its clinical application should be considered and made more widespread. 


\section{ACKNOWLEDGEMENTS}

We are grateful to Dr Can Liu and Qian Ding from the Department of Radiology, Jingzhou First People's Hospital, Hubei, for their excellent technical support during the RFA procedures.

\section{REFERENCES}

1. AACE/AAES Task Force on Primary Hyperparathyroidism. The American Association of Clinical Endocrinologists and the American Association of Endocrine Surgeons position statement on the diagnosis and management of primary hyperparathyroidism. Endocr Pract 2005; 11:49-54.

2. Tahim AS, Saunders J, Sinha P. A parathyroid adenoma: benign disease presenting with hyperparathyroid crisis. Case Report Med 2010; 2010:596185.

3. Lindekleiv H, Due J, Thuy L, Hansen TA, Nilsen PA. [Minimally invasive treatment of primary hyperparathyroidism.] Tidsskr Nor Laegeforen 2007; 127:1204-6. Norwegian.

4. Jeong $W K$, Baek JH, Rhim $\mathrm{H}$, et al. Radiofrequency ablation of benign thyroid nodules: safety and imaging follow-up in 236 patients. Eur Radiol 2008; 18:1244-50.

5. Tochio $\mathrm{M}$, Takaki $\mathrm{H}$, Yamakado $\mathrm{K}$, et al. A case report of 20 lung radiofrequency ablation sessions for 50 lung metastases from parathyroid carcinoma causing hyperparathyroidism. Cardiovasc Intervent Radiol 2010; 33:657-9.

6. Chan VO, McDermott S, Malone DE, Dodd JD. Percutaneous radiofrequency ablation of lung tumors: evaluation of the literature using evidence-based techniques. J Thorac Imaging 2011; 26:18-26.
7. Shin KY, Heo J, Kim JY, et al. A case of hemocholecyst associated with hemobilia following radiofrequency ablation therapy for hepatocellular carcinoma. Korean J Hepatol 2011; 17:148-51.

8. Carrafiello G, Laganà D, Mangini M, et al. Treatment of secondary hyperparathyroidism with ultrasonographically guided percutaneous radiofrequency thermoablation. Surg Laparosc Endosc Percutan Tech 2006; 16:112-6.

9. Adda G, Scillitani A, Epaminonda P, et al. Ultrasound-guided laser thermal ablation for parathyroid adenomas: analysis of three cases with a three-year follow-up. Horm Res 2006; 65:231-4.

10. Ahmad S, Jielani AA, Khan K, et al. Dual phase MIBI scintigraphy in diagnosis of parathyroid adenoma followed by ultrasound guided percutaneous alcoholic ablation. J Ayub Med Coll Abbottabad 2008; 20:149-51

11. Orlando A, Leandro G, Olivo M, Andriulli A, Cottone M. Radiofrequency thermal ablation vs. percutaneous ethanol injection for small hepatocellular carcinoma in cirrhosis: meta-analysis of randomized controlled trials. Am J Gastroenterol 2009; 104:514-24.

12. Bouza C, López-Cuadrado T, Alcázar R, Saz-Parkinson Z, Amate JM. Meta-analysis of percutaneous radiofrequency ablation versus ethanol injection in hepatocellular carcinoma. BMC Gastroenterology 2009; 9:31.

13. Akinci B, Demir T, Yener S, et al. Beneficial effect of endocrinologistperformed ultrasonography on preoperative parathyroid adenoma localization. Endocr Pract 2009; 15:17-23.

14. Lo CY, Lang BH, Chan WF, Kung AW, Lam KS. A prospective evaluation of preoperative localization by technetium-99m sestamibi scintigraphy and ultrasonography in primary hyperparathyroidism. Am J Surg 2007; 193:155-9.

15. Vazquez BJ, Richards ML. Imaging of the thyroid and parathyroid glands. Surg Clin North Am 2011; 91:15-32. 